\title{
The application of ferromagnetic additives to increase the hydraulic efficiency of main oil pipelines
}

\author{
Adelina Sagadeeva', Anvar Valeev ${ }^{2}$ \\ Department of Transport and storage of oil and Gas, Ufa State Petroleum Technological University, \\ Ufa, Russia \\ ${ }^{1}$ Corresponding author \\ E-mail: ${ }^{1}$ adel.sagadeeva@yandex.ru, ${ }^{2} a n v-v @ y a n d e x . r u$
}

Received 15 May 2021; accepted 3 June 2021

DOI https://doi.org/10.21595/lger.2021.22076

Check for updates

Copyright $(0) 2021$ Adelina Sagadeeva, et al. This is an open access article distributed under the Creative Commons Attribution License, which permits unrestricted use, distribution, and reproduction in any medium, provided the original work is properly cited.

\begin{abstract}
The article presents researches of ferromagnetic additives that can reduce the internal roughness of the pipeline, thereby reducing the hydraulic resistance. A setup has been developed for conducting experiments to determine the effectiveness of ferromagnetic additives. The results of experiments are presented, which showed that the additives allow increasing the volumetric flow rate of the pumped medium.
\end{abstract}

Keywords: oil product pipeline, hydraulic resistance, capacity, roughness, additives, volumetric flow rate, magnetic fluid.

\section{Introduction}

Pipeline transportation of oil and oil products has many advantages, but to move hydrocarbons over great distances, it is necessary to create high pressure in the pipe, which is achieved by the operation of mainline pumps, which consume a large amount of electricity. In general, a decrease in pressure losses along the length of the pipeline will increase their throughput and reduce electricity consumption, which will lead to a significant economic effect on the scale of the entire pipeline system of trunk transport.

When transporting oil in a pipeline, energy losses to overcome hydraulic frictional resistance directly depend on the coefficient of hydraulic resistance $\lambda$, the value of which is greatly influenced by the equivalent roughness of the inner surface of the pipe. Thus, by reducing the roughness value, it is possible to significantly reduce the energy losses of the pumped oil and increase the throughput of the oil product pipeline.

To reduce the hydraulic resistance in the pipeline, various types of anti-turbulent additives are actively used [1]. They have proven efficiency and are widely used, but they are expensive and require constant introduction into the pipeline.

It is urgent to study new types of additives, which can give an additional effect to increase the throughput and reduce the energy consumption of the pipeline system.

The study of ferromagnetic additives is promising.

\section{Ferromagnetic additives}

Ferromagnetic additives are a lubricating medium containing metal microparticles. Ferromagnetic microparticles will create a separating layer in the contact zone, which prevents the interaction of the medium with the wall. Such particles are able to change the microrelief of the contact zone, filling the microroughness of the friction surfaces [2].

The use of a ferromagnetic fluid as a lubricant is widely used in plain bearings between the shaft and the sleeve to reduce sliding frictional forces [3].

As practice shows, most plain bearings operate in hydrodynamic lubrication mode, where the viscosity of the lubricant plays an important role. During operation of the bearing in the friction unit, a significant increase in temperature occurs, the viscosity of the lubricant decreases, and as a result, the bearing capacity of the film thickness decreases [4]. All these processes lead to 
reduced bearing life. In order to increase the bearing capacity of the lubricant, a finely dispersed metal powder is added to it, which is able to increase the fluid's resistance to shear.

Also, for bearings and various lubrication units, magnetic-liquid sealants are actively used [5], the principle of operation of which is to form and maintain an annular layer of magnetic fluid on a rotating or moving shaft in the area of a strong magnetic field formed by installed magnets. wear of shafts.

The same principle of reducing hydraulic resistance can be applied in pipeline transport through the use of ferromagnetic additives.

After in-line diagnostics and cleaning the inner surface of the pipe using various diagnostic and cleaning devices, the pipeline has residual magnetization. In addition, due to the installation of magnets or a magnetic film on the pipeline, it is possible to create an additional magnetic field inside the oil product pipeline. The essence of the method is that during the transportation of the medium, ferromagnetic microparticles will be attracted to the inner surface of the magnetized pipe, filling the microroughnesses of the pipeline and reducing the roughness.

The aim of the authors' research is to study ferromagnetic additives, the nanoparticles of which are able to change the microrelief of the contact zone and reduce the value of hydraulic resistance.

The objective of the experimental studies given below is to test the fundamental possibility of reducing the hydraulic resistance in the pipeline due to ferromagnetic additives.

\section{Experimental part}

To carry out experiments to determine the effectiveness of ferromagnetic additives, a setup was designed, shown in Fig. 1.

The experimental setup consists of two tanks with a volume of 40 liters, interconnected by a pipeline $0.62 \mathrm{~m}$ long and $1 / 2$ in diameter. A copper wire winding with a pitch of $1 \mathrm{~mm}$ is applied to the pipeline, which is connected to a power source - a battery with a voltage of $9 \mathrm{~V}$. Since the side walls of the tanks have a slope, the tanks were additionally calibrated.

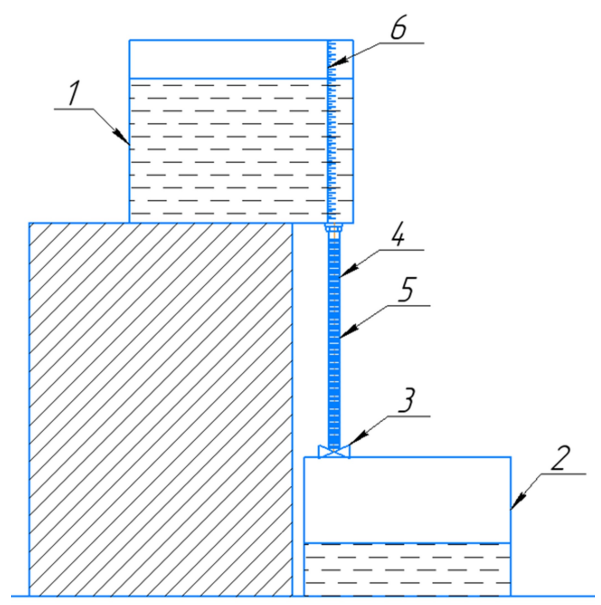

a)

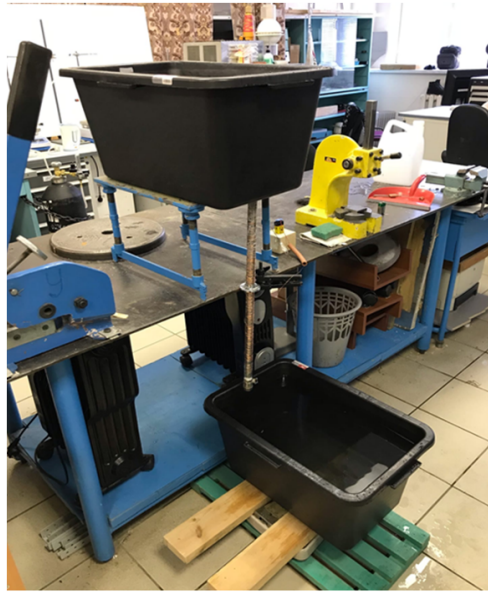

b)

Fig. 1. Installation for conducting an experiment to determine the effectiveness of ferromagnetic additives: a) installation diagram; b) general view of the installation: 1 - tank No. $1 ; 2-\operatorname{tank}$ No. 2 ; 3 - crane; 4 - pipeline; 5 - copper wire winding; 6 - scale

In the course of the work, 14 experiments were carried out, 7 of which were carried out without the use of ferromagnetic additives, the rest - with additives. The experiments themselves were carried out at room temperature, equal to $20^{\circ} \mathrm{C}$, as follows. Water was poured into the upper tank of the installation in a volume of 36 liters at first without additives. Next, a valve was opened, installed on a fixed and vertically located pipeline. Afterwards, the change in the liquid level in 
the tank, that is, the volume of water that passed through the pipe in $100 \mathrm{~s}$, was determined by a scale installed inside the upper tank of the installation. In the same sequence, experiments were carried out with ferromagnetic additives, the volume of which was $0.5 \mathrm{ml}$.

The results of experiments without the use of additives are presented in Table 1.

Table 1. The results of experiments without the use of additives

\begin{tabular}{|c|c|c|}
\hline Experiment No. & Time, $\mathrm{s}$ & Volume flow, $\mathrm{m}^{3} / \mathrm{h}$ \\
\hline 1 & 100,23 & 1,064 \\
\hline 2 & 100,47 & 1,069 \\
\hline 3 & 100,33 & 1,065 \\
\hline 4 & 100,32 & 1,066 \\
\hline 5 & 100,33 & 1,075 \\
\hline 6 & 100,12 & 1,065 \\
\hline 7 & 100,09 & 1,061 \\
\hline Average & 100,35 & 1,067 \\
\hline
\end{tabular}

Experiments without the use of ferromagnetic additives have shown that the average volumetric flow rate of water passed through the pipe for $(100.3 \pm 0.2) \mathrm{s}$, taking into account the error, is $Q=(1.067 \pm 0.009) \mathrm{m}^{3} / \mathrm{h}$.

The results of experiments using ferromagnetic additives are presented in Table 2.

Table 2. Results of experiments using ferromagnetic additives

\begin{tabular}{|c|c|c|}
\hline Experiment No. & Time, $\mathrm{s}$ & Volume flow, $\mathrm{m}^{3} / \mathrm{h}$ \\
\hline 1 & 100,33 & 1,113 \\
\hline 2 & 100,51 & 1,129 \\
\hline 3 & 100,59 & 1,124 \\
\hline 4 & 100,53 & 1,124 \\
\hline 5 & 100,63 & 1,128 \\
\hline 6 & 100,76 & 1,122 \\
\hline 7 & 100,47 & 1,130 \\
\hline Average & 100,54 & 1,124 \\
\hline
\end{tabular}

Experiments with the use of additives showed that the average volumetric flow rate of water passed through the pipe in $(100.5 \pm 0.2) \mathrm{s}$, taking into account the error, is $Q=(1.124 \pm 0.019) \mathrm{m}^{3} / \mathrm{h}$.

\section{Results}

The results of all experiments are shown in Fig. 2.

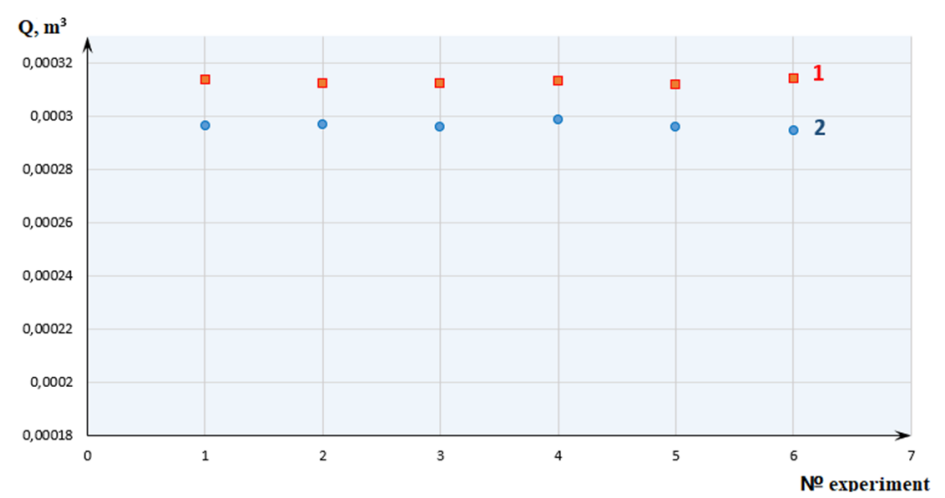

Fig. 2. Change in volumetric flow rate of water: 1 -with ferromagnetic additives; 2 - no additives 
At a concentration of ferromagnetic additives $C=0.0014 \%$, it was possible to increase the volumetric flow rate of water passing through the pipe for $100 \mathrm{~s}$ by $(6 \pm 2) \%$.

Thus, it can be concluded that ferromagnetic additives make it possible to: create a separating layer between the pumped medium and the pipe wall, reduce the equivalent roughness of the inner surface of the pipeline, and also increase the volumetric flow rate and throughput of oil product pipelines.

The results of the experiments carried out also allow us to assume that with an increase in the concentration of ferromagnetic additives, their efficiency will increase, and, as a consequence, the coefficient of hydraulic resistance will decrease.

The dependence of the hydraulic resistance coefficient on the concentration of the additive for the pipe used in the experiment is shown in Fig. 3. Without the use of additives, the coefficient $\lambda$ is 0.0227 . When adding additives in a volume of $0.5 \mathrm{ml}$ to the pipe, the $\lambda$ value becomes equal to 0.0204 , which is more than $10 \%$ lower than the initial value. Theoretically, with an increase in the concentration of additives, the hydraulic resistance coefficient will tend to zero. So in the case when the equivalent roughness of the pipe becomes equal to zero $\left(k_{e}=0\right)$, the value of $\lambda$ will be approximately equal to 0.0189 .

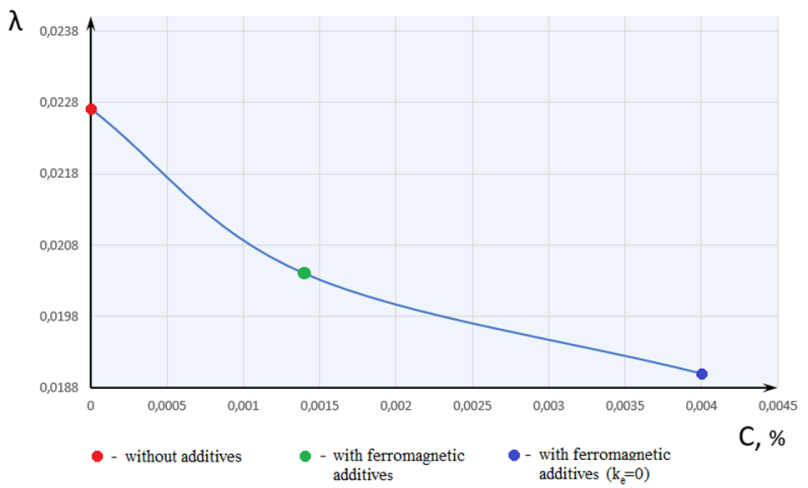

Fig. 3. Dependence of the hydraulic resistance coefficient of the experimental pipe on the concentration of ferromagnetic additives

If we transfer the obtained values to a real pipeline, for example, to the Tikhoretsk-Novorossiysk oil product pipeline with a length of $244 \mathrm{~km}$ and a diameter of $530 \mathrm{~mm}$, through which diesel fuel with a density of $850 \mathrm{~kg} / \mathrm{m}^{3}$ is transported, then the dependence of the hydraulic resistance coefficient on the concentration of a ferromagnetic additive will be a curve, shown in Fig. 4.

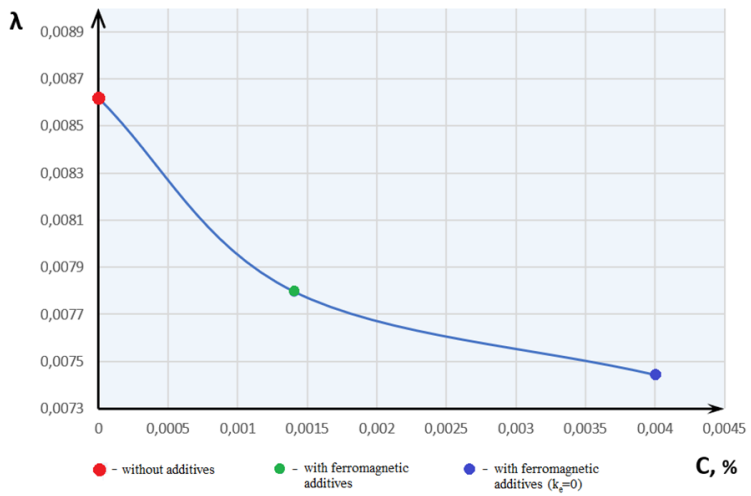

Fig. 4. Dependence of the hydraulic resistance coefficient of the Tikhoretsk-Novorossiysk oil product pipeline on concentration ferromagnetic additives 
Consequently, the use of ferromagnetic additives will allow when pumping oil products to move from the area of square friction, where hydraulic losses depend on the degree of pipe roughness, to the area of hydraulically smooth pipes, where the coefficient of hydraulic resistance does not depend on the roughness.

\section{Conclusions}

So, the fundamental possibility of reducing the coefficient of hydraulic resistance in the pipeline due to the use of a ferromagnetic additive was proved, and the tasks of the next studies will be to study the dependence of the equivalent roughness on the concentration of ferromagnetic particles. It is also necessary to consider whether the additive affects the quality of the oil, respectively, if there is a need to capture it, for example, in filters at the station entrance due to magnetic traps. It is also important to study the stability of the layer of ferromagnetic particles at the wall-oil product interface, including whether they are entrained by the flow, whether there is a need to re-add the additive.

Based on the studies carried out, it can be concluded that ferromagnetic additives are promising for use in oil product pipelines, since they can significantly reduce the coefficient of hydraulic resistance in the pipeline, and, consequently, reduce the energy consumption of pumping units.

\section{References}

[1] Konovalov K. B., et al. Comparative study of the action of anti-turbulent additives for hydrocarbon fluids. Brief Communications on Physics, Vol. 12, 2015, p. 36-42.

[2] Ye Krasilnikova D. Influence of the Viscosity of the Carrier Fluid on the Magnetic Properties of Ferrofluids. 2015, p. 202-213.

[3] Perekrestov A. P., et al. The mechanism of action of an antiwear additive on a magnetic basis. Vestnik AGTU, Vol. 3, 2008, p. 47-50.

[4] Orlov D. V., et al. Magnetic Liquids in Mechanical Engineering. Mechanical Engineering, 1993, p. 268.

[5] Kurapov P. A. On the mechanism of the lubricating action of magnetic fluids. 5th All-Union Conference on Magnetic Fluids, 1988, p. 154-155.

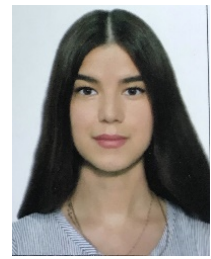

Adelina Sagadeeva received Bachelor degree in Ufa State Petroleum Technological University, Ufa, Russia. Her current research interests include magnetic fluids, increasing the hydraulic efficiency of pipelines.

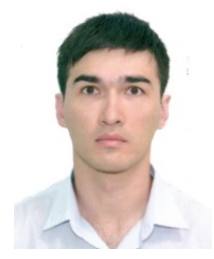

Anvar Valeev is Candidate of Engineering Science, Associate Professor of Department "Oil and Gas Transport and Storage" of Ufa State Petroleum Technological University, leading researcher. Anvar Valeev, a specialist in the field of vibration protection, diagnostics, condition monitoring of industrial equipment, also systems with quasi-zero stiffness, transport and storage of oil and gas. He is the author of over 130 scientific publications and the co-author of textbooks, monograph and patents. Anvar Valeev is supervisor of number of scientific grants. He presented the main results of scientific research at the international conferences in St. Petersburg, Moscow, Samara, Ufa, Shaghai (China), Seoul (South Korea), Athens (Greece), London (United Kingdom), Hiroshima (Japan), Rome (Italy). 